\title{
Preparation and Characterization of Core/Shell-type Ag/Chitosan Nanoparticles with Antibacterial Activity
}

\author{
Yue Lin," Wang Jing, Pan Kang, Zhang Xiaoming, Wang Zhouping, and Xia Wenshui \\ State Key Laboratory of Food Science and Technology and School of Food Science and Technology, Jiangnan University, \\ Wuxi, 214122, PR China. ${ }^{*}$ E-mail: yuelin@jiangnan.edu.cn \\ Received December 14, 2010, Accepted February 15, 2011
}

\begin{abstract}
Making use of chitosan (CS) and ethylenediaminetetraacetic acid (EDTA) as a reaction system, CS-EDTA nanoparticles were synthesized through a facile counterion complex coacervation method. $\mathrm{Ag}^{+}$could enter porous CS nanoparticles synthesized with this method, allowing Ag nanoparticles within chitosan nanoparticles were synthesized by reducing silver nitrate with chitosan. Because of the noncovalent interaction between CS and EDTA, the EDTA could be easily removed via dialysis against water, and pure core/shell-type $\mathrm{Ag} / \mathrm{CS}$ nanoparticles could be obtained. The nanoparticles showed higher antibacterial activity toward $E$. coli than the active precursor Ag nanoparticles and CS.
\end{abstract}

Key Words : Chitosan, Nanocomposite, Antibacterial activity

\section{Introduction}

Chitosan (CS), obtained by extensive deacetylation of chitin isolated from crustacean shell, is a natural polysaccharide with many useful features such as biocompatibility, biodegradability and antibacterial properties. It, as well as its derivatives, has great potential applications in such areas as biotechnology, biomedicine, and food technology. ${ }^{1}$

Silver-containing materials have long been recognized as effective antimicrobial agents. Previous studies have demonstrated that both ionic and metallic silver exhibit antimicrobial activities, ${ }^{2,3}$ although the mechanism of the inhibitory action of silver on microbes is still not fully understood. It has been proposed that silver ions, in interaction with disulfide or sulfhydryl groups of enzymes, should cause structural changes that lead to disruption of metabolic processes and even death of cells. ${ }^{4,5}$ The antibacterial action of $\mathrm{Ag}$ nanoparticles might be associated with the release of $\mathrm{Ag}^{+} .^{+-9}$ In addition, it has been suggested that the property may also be related to membrane damage as a result of free radicals that are derived from the surface of the nanoparticles. ${ }^{10}$ This property might be dependent on the size and the shape of $\mathrm{Ag}$ nanoparticles. ${ }^{11}$

For economical and efficient purpose, composite materials incorporating Ag nanoparticles have been developed and tested, one of which is chitosan-Ag composite whose antibacterial activity has been widely explored. To improve antimicrobial activity, various sorts of chitosan-Ag composites have been prepared in recent years. For example, Du et al. synthesized CS nanoparticles loaded with $\mathrm{Ag}^{+}$and probed into their antibacterial activity, finding a significant enhancement of the activity. ${ }^{12}$ Sanpui $P$ et al. reported that a novel chitosan-Ag-nanoparticle composite was synthesized and demonstrated a significantly higher antimicrobial activity than its components at the respective concentrations. ${ }^{13}$ Zhao et al. prepared Ag-chitosan composite nanoparticles and researched their antibacterial activity, where CS was a stablizing agent and $\mathrm{Vc}$ as a reducing agent for $\mathrm{Ag}^{+}$to form $\mathrm{Ag}$ nanoparticles. ${ }^{14}$ Wei et al. synthesized CS-based Ag nanoparticles and demonstrated their high antibacterial activity against Gram-positive and Gram-negative bacteria. ${ }^{15}$ Madhuchanda B et al. reported synergy in antimicrobial activity of a CS-Ag nanoparticle composite in the presence of molecular iodine. ${ }^{16}$ To the best of our knowledge, no report has so far dealt with the preparation of individual core/shell-type $\mathrm{Ag} / \mathrm{CS}$ nanoparticles, though there have been reports concerning Ag-chitosan composite nanoparticles. ${ }^{17,18}$ The present study aims to prepare highly dispersible core/ shell-type $\mathrm{Ag} / \mathrm{CS}$ nanoparticles and test their antibacterial activity toward E. coli.

\section{Experimental}

Materials. CS was purchased from Golden-Shell Biomedical Company (Zhejiang, China) and used without further purification. The degree of deacetylation and the molecular weight were around $85 \%$ and 5000 as determined by elemental analysis and viscometric method respectively. EDTA, glutaraldehyde (GA) and $\mathrm{AgNO}_{3}$ (Shanghai Chemical Reagent company) were used as received. All other ingredients were of analytical grade unless otherwise stated. Escherichia coli ATCC 8739 was selected as indicators of experimental bacteria. Luria Bertani (LB) broth was used as a growing medium. The bacteria were grown aerobically in LB broth at $37{ }^{\circ} \mathrm{C}$ for $24 \mathrm{~h}$.

Preparation of Core/Shell-type Ag/CS Nanoparticles. The CS-EDTA composite nanoparticles were prepared based on the counterion complex coacervation method. A brief outline of the method is as follows. $100 \mathrm{mg}$ of CS was dissolved in $20 \mathrm{~mL}$ of water. $34 \mathrm{mg}$ of EDTA was added to the CS aqueous solution and heated until dissolution. Then, ethanol was added dropwise to the system with vigorous 
stirring, and the clear solution turned opalescent when the ethanol attained $32 \mathrm{~mL}$, implying the formation of colloidal particles. After that, $70 \mu \mathrm{L}$ of GA solution (25\%) was used to cross-link the nanoparticles obtained at room temperature for $4 \mathrm{~h}$. The cross-linked nanoparticles were purified using centrifugation, followed by redispersing the sediment into ethanol solution $(40 \% \mathrm{v} / \mathrm{v})$.

The CS-EDTA nanoparticles loaded with $\mathrm{Ag}^{+}$were obtained by adding of $50 \mu \mathrm{L}$ of $\mathrm{AgNO}_{3}$ solutions $(1 \% \mathrm{w} / \mathrm{w})$ into the $2 \mathrm{~mL}-\mathrm{CS}-\mathrm{EDTA}$ suspension $(0.25 \% \mathrm{w} / \mathrm{v})$ and continuously stirring for $10 \mathrm{~min}$ at room temperature. The mixture, after reacting for $5 \mathrm{~h}$ at room temperature, was left slowly cooled and dialyzed against pure water for $24 \mathrm{~h}$ to remove the EDTA molecules.

Characterization. The mean diameter and size distribution of the CS-EDTA composite nanoparticles were determined by Dynamic Light Scattering (DLS) Analysis using a Zetasizer Nano ZS instrument (Malvern Instruments Corporation). The UV-visible absorption spectra were recorded on a Shimadzu UV3100 spectrophotometer (Shimadzu, Japan). The samples were dried in a freeze-dryer prior to FT-IR analyses. FT-IR spectra were collected using a Bruke IFS66V vacuum-type spectrometer (Bruke, Germany). The morphology of the CS-EDTA composite nanoparticles and the AgChitosan composite nanoparticles were observed using transmission electron microscopy (TEM) (JEM-2100, JEOL, Japan). For TEM observations, 1 drop of properly diluted sample was placed on a copper grid and air dried before examination.

\section{Results and Discussion}

Being a cationic biopolymer bearing amino groups, CS can undergo counterion complex coacervation in an aqueous solution of multivalent anionic substance including sodium tripolyphosphate or EDTA to form colloidal aggregate nanoparticles. Size distribution is an essential parameter for nanoparticles. As shown in Figure 1, the CS-EDTA composite nanoparticles achieve a mean diameter of $90 \mathrm{~nm}$ with a

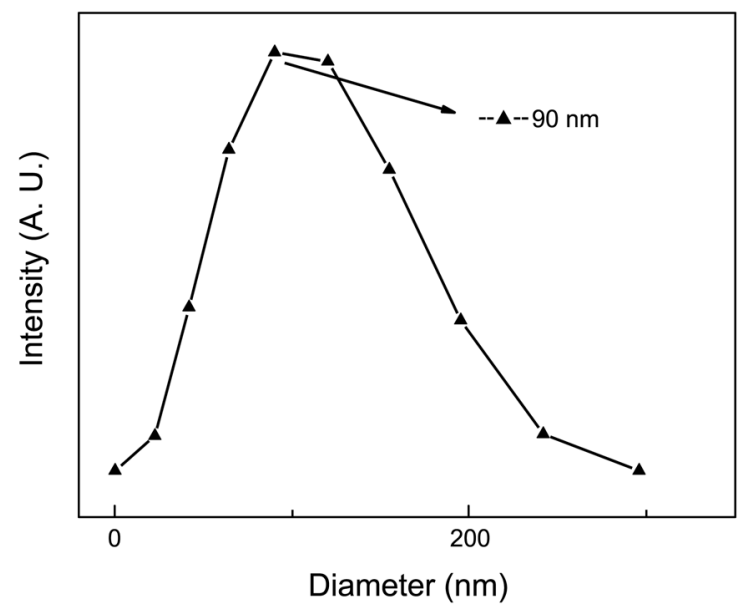

Figure 1. Size distributions of CS-EDTA composite nanoparticles in CS and EDTA mixed solution as the value of ethanol attained 32 $\mathrm{mL}$.

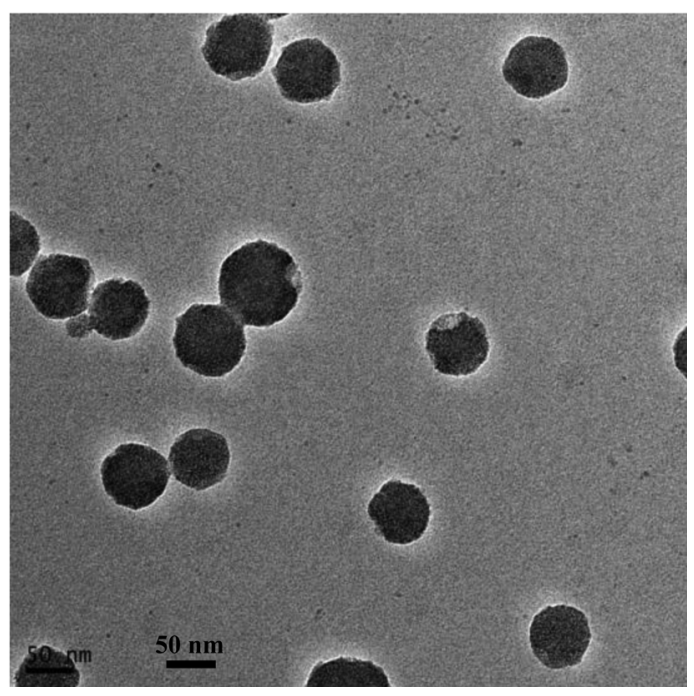

Figure 2. TEM of the CS-EDTA composite nanoparticles.

narrow size distribution. In Figure 2, the cross-linked composite nanoparticles show a typical $90-\mathrm{nm}$ size distribution, as determined by DLS. It can be noticed that the nanoparticles had a spherical shape and a nearly uniform size that was slightly smaller than the DLS result because of the dehydration of the nanoparticles.

Because the CS-EDTA composite nanoparticles are porous, EDTA can be removed by dialysis against water. $\mathrm{Ag}^{+}$ion, which chelates with $-\mathrm{NH}_{2}$ of chitosan, can enter CS nanoparticles and be stabilized by CS. In the process of preparing, $\mathrm{Ag}^{+}$ion was converted into $\mathrm{Ag}$ nanoparticles using the CS molecules as the reductant. ${ }^{15}$ The color of the solution turned from yellow to black-red, implying that Ag salt had been reduced and Ag nanoparticles had formed, which was verified by UV and TEM analyses. Under the same conditions neither GA nor ethanol can reduce $\mathrm{AgNO}_{3}$ to $\mathrm{Ag}$. Figure 3 shows the UV-vis absorption spectra of the CS nanoparticles, the CS nanoparticles loaded with $\mathrm{Ag}^{+}$, the $\mathrm{Ag}$ nanoparticles, and the core/shell-type $\mathrm{Ag} / \mathrm{CS}$ nanoparticles.

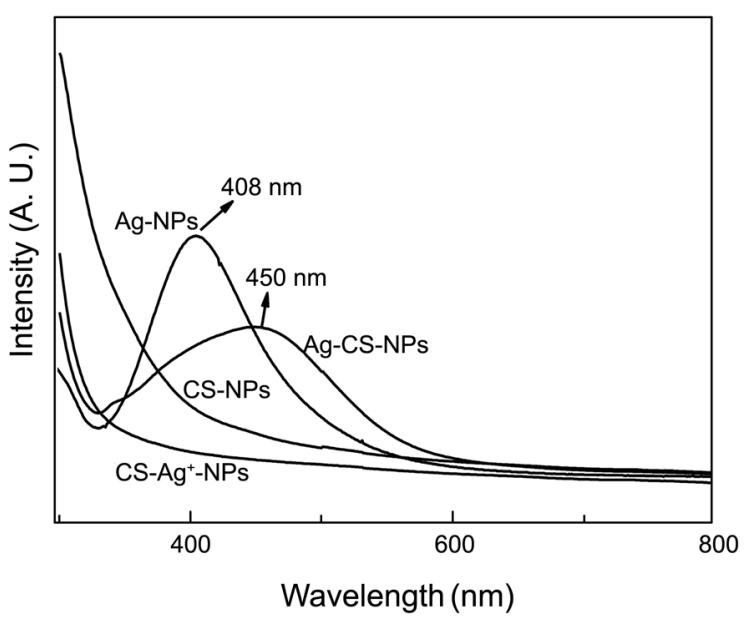

Figure 3. UV-vis spectra of CS nanoparticles (CS-NPs), CS nanoparticles loaded $\mathrm{Ag}^{+}\left(\mathrm{CS}-\mathrm{Ag}^{+}-\mathrm{NPs}\right), \mathrm{Ag}$ nanoparticle (Ag$\mathrm{NPs}$ ), and core/shell-type Ag/CS nanoparticles (Ag-CS-NPs). 

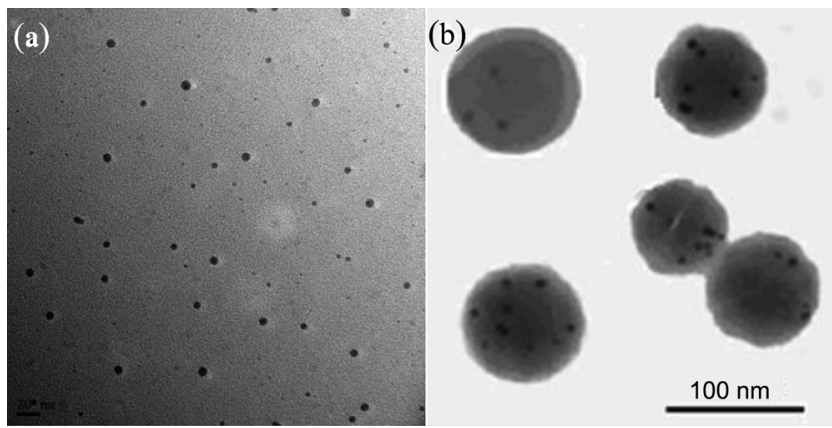

Figure 4. TEM images of Ag-NPs (a) and Ag-CS-NPs (b).

It is obvious that neither the CS nanoparticles nor the CS nanoparticles loaded with $\mathrm{Ag}^{+}$can indicate absorbance between a $300-\mathrm{nm}$ and $900-\mathrm{nm}$ wavelength. The spectrum for the suspension of the $\mathrm{Ag}$ nanoparticles has a surface plasmon absorption at $408 \mathrm{~nm}$ arising from the nanoparticles, and the color of the suspension was yellow, which is characteristic of $\mathrm{Ag}$ colloidal nanoparticles. A similar surface plasmon absorption is observed for the suspension of the core/shell-type $\mathrm{Ag} / \mathrm{CS}$ nanoparticles at a wavelength longer than that for the suspension of the Ag nanoparticles. This absorption shift is caused by a different refractive index of the CS shell and strongly suggests a complete coverage of the Ag nanoparticles with CS. ${ }^{19}$

Figure 4 shows the typical TEM images of the Ag nanoparticles and the core/shell-type $\mathrm{Ag} / \mathrm{CS}$ nanoparticles. In Figure 4(a) the Ag nanoparticles are spherical, with a size of $5 \mathrm{~nm}$ or so. After the reaction of $\mathrm{AgNO}_{3}$ reduction, as shown in Figure 4(b), the well-dispersed core/shell-type $\mathrm{Ag} / \mathrm{CS}$ nanoparticles are obtained in the darker region being 5-nm $\mathrm{Ag}$ nanoparticles and in the lighter out-ring being CS. The contrast between the core and shell region is caused by the different electron penetration efficiency on the Ag nanoparticles and the CS nanoparticles. In these core/shell-type $\mathrm{Ag} / \mathrm{CS}$ nanoparticles, about $5 \mathrm{~nm}$ of the $\mathrm{Ag}$ nanoparticles are encapsulated within a CS layer (shell).

Figure 5 presents the FT-IR of CS, EDTA, the CS-EDTA nanoparticles, the core/shell-type $\mathrm{Ag} / \mathrm{CS}$ nanoparticles at the wave number ranging from 4000 to $400 \mathrm{~cm}^{-1}$, which provides further information on the structure of the nanoparticles synthesized. The absorption bands at 3430 and $1630 \mathrm{~cm}^{-1}$ or so, as observed in all spectra, are attributed to the stretching mode of hydroxyl. ${ }^{20,21}$ The absorption peak of the carbonyl group at about $1690 \mathrm{~cm}^{-1}$ in EDTA shifts to $1630 \mathrm{~cm}^{-1}$ in the spectrum of the CS-EDTA nanoparticles and the core/shelltype $\mathrm{Ag} / \mathrm{CS}$ nanoparticles, as the carboxyls in EDTA molecules are ionized and overlapped with bending vibration of hydroxyl. ${ }^{22}$ At the same time, the amino band (at $1540 \mathrm{~cm}^{-1}$ ) in the spectrum of pure chitosan disappears in the CS-EDTA nanoparticles and the core/shell-type Ag/CS nanoparticles, suggesting that the amino groups are in their protonated state whose absorption peak (around $1620 \mathrm{~cm}^{-1}$ ) may be overlapped by that of the hydroxyl in the nanoparticles (1630 $\mathrm{cm}^{-1}$ ). These changes in the FT-IR spectrum indicate that the carboxyl groups of EDTA molecules in the ionized state

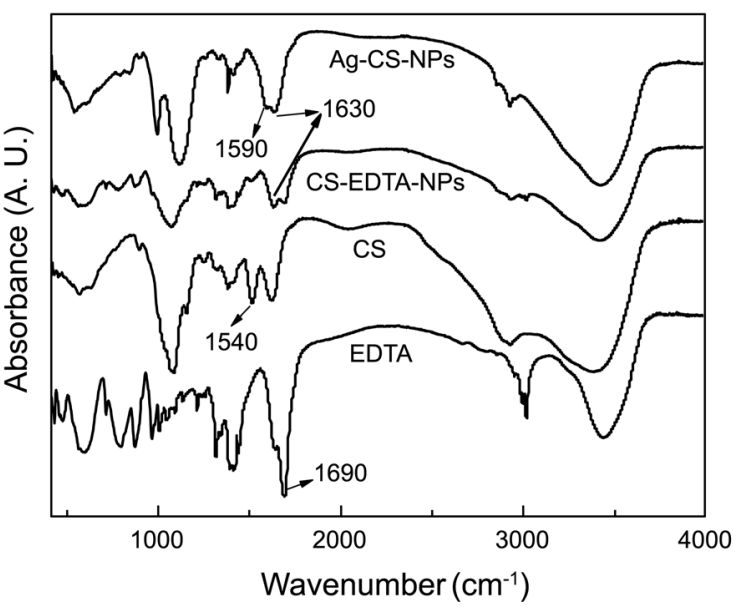

Figure 5. FT-IR spectra of EDTA, CS, CS-EDTA-NPs and Ag-CSNPs.

form ionic bonds, with the protonated amino groups of chitosan forming the composite CS-EDTA nanoparticles via counterion complex coacervation. In the spectrum of the core/shell-type $\mathrm{Ag} / \mathrm{CS}$ nanoparticles, the amino band at 1540 $\mathrm{cm}^{-1}$ shifts to a higher wavenumber with a shoulder peak arriving at $1590 \mathrm{~cm}^{-1}$, which implies an interaction between the amine groups of $\mathrm{CS}$ and the $\mathrm{Ag}$ nanoparticles present in the core/shell-type $\mathrm{Ag} / \mathrm{CS}$ nanoparticles. ${ }^{23}$

As discussed for synthesis of CS nanoparticles, ${ }^{22,24,25}$ the current assembly of CS nanoparticles occurs through electrostatic attraction interactions, i.e., chitosan as a polycation, and EDTA as an anionic compound. Yet, the electrostatic attraction is insufficient to form clusters when the two solutions are mixed. Therefore, we used a nonsolvent-aided counterion complexation method, which may promote the interaction between chitosan and EDTA. Ethanol is introduced in our experiment as a nonsolvent. Addition of ethanol leads to the closeness of the chitosan chains to EDTA molecules because of the desolvation of chitosan, resulting in formation of nanoparticles. EDTA can be easily removed via dialysis against water as a result of the noncovalent interaction between CS and EDTA. By diffusion, $\mathrm{Ag}^{+}$in the CS nanoparticles can be reduced to be Ag nanoparticles by CS. As is well known, the good affinity of CS is a favorable factor to form core/shell-type $\mathrm{Ag} / \mathrm{CS}$ nanoparticles.

The antibacterial properties of the core/shell-type $\mathrm{Ag} / \mathrm{CS}$ nanoparticles are investigated by inhibition testing. Nutrient agar was poured into disposable sterilized Petri dishes and allowed to solidify. $200 \mathrm{~mL}$ of $E$. coli bacterial water (about $10^{6} \mathrm{CFU} / \mathrm{mL}$ ) was streaked uniformly over each plate. Samples of the CS nanoparticles $(1 \% \mathrm{~W} / \mathrm{V})$, the $\mathrm{Ag}$ nanoparticles $(0.06 \% \mathrm{~W} / \mathrm{V})$, and the core/shell-type $\mathrm{Ag} / \mathrm{CS}$ nanoparticles $(1 \% \mathrm{~W} / \mathrm{V})$ were suspended respectively to reach the appropriate concentration. Pieces $(1 \mathrm{~cm} \times 1 \mathrm{~cm})$ of conventional round filter paper, a CS nanoparticle sheet, a Ag nanoparticle sheet, and the core/shell-type $\mathrm{Ag} / \mathrm{CS}$ nanoparticles sheet, were soaked in the solutions and sterilized respectively, and gently placed on the solidified agar gel in separate Petri dishes. Then, the plates were incubated at $37^{\circ} \mathrm{C}$ for $24 \mathrm{~h}$. 


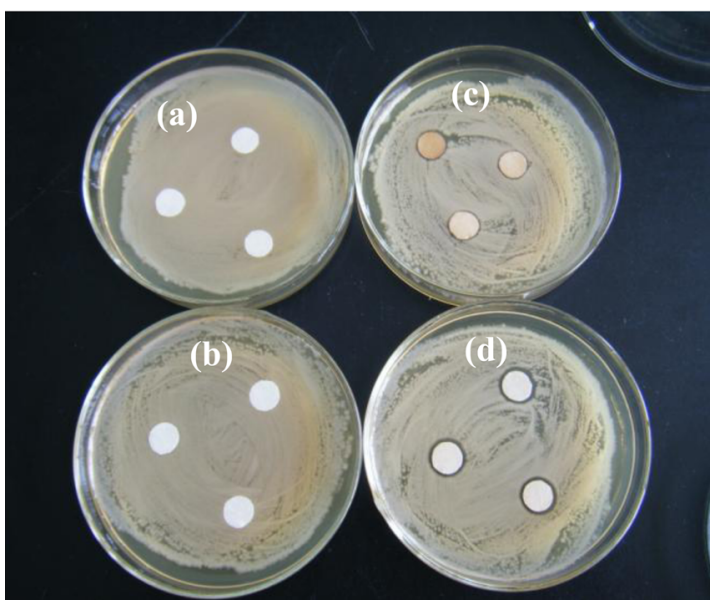

Figure 6. The results of inhibition testing. (a), (b), (c), (d) conventional filter paper, CS NPs sheet, Ag NPs sheets and Ag-CS-NPs sheet, respectively.

The colour of the Ag nanoparticle sheet was yellow because it absorbed the Ag nanoparticles. As shown in Figure 6, an obvious zone of inhibition appeared around the Ag nanoparticle sheet and around the core/shell-type $\mathrm{Ag} / \mathrm{CS}$ nanoparticles sheet. Yet, for the core/shell-type $\mathrm{Ag} / \mathrm{CS}$ nanoparticles sheet, the inhibition zone is larger than that of the Ag nanoparticle sheet, which shows the core/shell-type Ag/ CS nanoparticles may have a better antibacterial property than the $\mathrm{Ag}$ nanoparticles. Meanwhile no growth was present on the filter paper (Fig. 6(a)) and on the CS nanoparticle sheet (Fig. 6(b)). The results clearly indicate the high antibacterial properties of the core/shell-type $\mathrm{Ag} / \mathrm{CS}$ nanoparticles.

The high antibacterial activity of the core/shell-type Ag/ CS nanoparticles might be ascribed to the synergistic effects of Ag nanoparticles and CS, as elaborated as follows. On the one hand, the Ag nanoparticles exhibit the effective antimicrobial activity as a result of their strong binding to the electrondonating groups in the bacterial cells. Once the core/ shell-type $\mathrm{Ag} / \mathrm{CS}$ nanoparticles release the Ag nanoparticles into the pathogenic environment, the $\mathrm{Ag}$ nanoparticles will attach themselves to the surface of the bacterial cells or even penetrate in, resulting in the antimicrobial activity. On the other hand, the CS can also make some contribution to the antibacterial activity of the core/shell-type $\mathrm{Ag} / \mathrm{CS}$ nanoparticles. The bactericidal activities of the CS, as reported in previous works, ${ }^{26}$ enables it to catch the surrounding bacteria through the strong ionic interaction with the cytoplasmic membrane of the bacteria. Once the CS captures the bacteria, the cell membrane remnants are likely to be adsorbed on the CS surface. Yet the core/shell-type Ag/CS nanoparticles can still release the Ag nanoparticles into the pathogenic environment, ${ }^{27}$ leading to the highly efficient antibacterial activity of the nanocomposites. It can be reasonably presumed that $\mathrm{Ag}$ ions or $\mathrm{Ag}$ nanoparticles from the core/shell-type $\mathrm{Ag} / \mathrm{CS}$ nanoparticles could be released through porous CS shell, and that such a structure will benefit prolonging the release time of $\mathrm{Ag}$ ions or $\mathrm{Ag}$ nanoparticles and preserving the sustained antibacterial behavior.

\section{Conclusions}

The core/shell-type $\mathrm{Ag} / \mathrm{CS}$ nanoparticles with a novel structure and composition are investigated to determine their antimicrobial properties. First of all, the aforementioned core/shell-type $\mathrm{Ag} / \mathrm{CS}$ nanoparticles consisting of chitosan and Ag nanoparticles can be prepared based on a two-step reaction. The first step is the preparation of CS-EDTA nanoparticles through a counterion complex coacervation method via electrostatic attraction reaction. The second step is that $\mathrm{Ag}$ ion within CS-EDTA nanoparticles via diffusion is reduced to $\mathrm{Ag}$ nanoparticles with chitosan as the reductant. EDTA can be easily removed by dialysis against water and then the core/shell-type $\mathrm{Ag} / \mathrm{CS}$ nanoparticles are obtained. The inhibition testing reveals the core/shell-type $\mathrm{Ag} / \mathrm{CS}$ nanoparticles show highly potent antibacterial activity against E. coli.

This work integrates nanotechnology into bacteriology, leading to possible advances in the formulation of new types of bactericides. Despite this, future studies on the biocidal influence of this nanomaterial on other Gram-positive and Gram-negative bacteria are necessary in order to fully evaluate its possible use as a new bactericidal material.

Acknowledgments. This work is financially supported by the Fundamental Research Funds for the Central Universities (JUSRP30903). Author is grateful to Professor Le Guowei for excellent technical assistance for the inhibition testing of nanoparticles synthesized.

\section{References}

1. Chien, P. J.; Seu, F.; Yang, F. H. Journal of Food Engineering 2007, 78, 225.

2. Holt, K. B.; Bard, A. J. Biochemistry 2005, 44, 13214.

3. Lok, C. N.; Ho, C. M.; Chen, R.; He, Q. Y.; Yu, W. Y.; Sun, H. Z. et al. Journal of Proteome Research 2006, 5, 916.

4. Butkus, M. A.; Edling, L.; Labare, M. P. Journal of Water Supply: Research and Technology - Aqua 2003, 52, 407.

5. Feng, Q. L.; Wu, J.; Chen, G. Q.; Cui, F. Z.; Kim, T. N.; Kim, J. O. Journal of Biomedical Materials Research 2000, 52, 662.

6. Panacek, A.; Kvitek, L.; Prucek, R.; Kolar, M.; Vecerova, R.; Pizurova, N. et al. The Journal of Physical Chemistry B 2006, 110,16248 .

7. Baker, C.; Pradhan, A.; Pakstis, L.; Pochan, D. J.; Shah, S. I. Journal of Nanoscience and Nanotechnology 2005, 5, 244.

8. Cho, K. H.; Park, J. E.; Osaka, T.; Park, S. G. Electrochim Acta 2005, $51,956$.

9. Sondi, I.; Salopek-Sondi, B. Journal of Colloid and Interface Science 2004, 275, 177.

10. Kim, J. S. K.; Kuk, E.; Yu, K. N.; Kim, J. H.; Park, S. J.; Lee, H. J. et al. Nanomedicine: Nanotechnology, Biology and Medicine 2007, 3, 95.

11. Pal, S.; Tak, Y. K.; Song, J. M. Applied and Environmental Microbiology 2007, 73, 1712.

12. Du, W. L.; Niu, S. S.; Xu, Y. L.; Xu, Z. R.; Fan, C. L. Carbohydrate Polymers 2009, 75, 385.

13. Sanpui, P.; Murugadoss, A.; Prasad, P. V. D.; Ghosh, S. S.; Chattopadhyay, A. International Journal of Food Microbiology 
2008, 124, 142.

14. Cao, X. L.; Cheng, C.; Ma, Y. L.; Zhao, C. S. J. Mater Sci.: Mater Med. 2010, 21, 2861.

15. Wei, D. W.; Sun, W. Y.; Qian, W. P.; Ye, Y. Z.; Ma, X. Y. Carbohydrate Research 2009, 344, 2375.

16. Banerjee, M.; Mallick, S.; Paul, A.; Chattopadhyay, A.; Ghosh, S. S. Langmuir 2010, 26, 5901.

17. Twu, Y. K.; Chen, Y. W.; Shih, C. M. Powder Technology 2008, $185,251$.

18. Murugadoss, A.; Chattopadhyay, A. Nanotechnology 2008, 19, 015603.

19. Empleton, A.C.; Pietron, J. J.; Murray, R. W.; Mulvaney, P. The Journal of Physical Chemistry B 2000, 104, 564.

20. Dutoit, D. C. M.; Schmeider, M.; Baiker, A. Journal of Catalysis
1995, 153, 165.

21. Rubio, J.; Oteo, J. L.; Villegas, M.; Duran, P. Journal of Materials Science 1997, 32, 643.

22. Guo, R.; Zhang, L.; Zhu, Z.; Jiang, X. Langmuir 2008, 24, 459.

23. Wei, D. W.; Qian, W. P. Colloids and Surfaces B-Biointerfaces 2008, 62, 136.

24. Bodnar, M.; Hartmann, J. F.; Borbely, J. Biomacromolecules 2005, 6, 2521.

25. Yu, S. Y.; Hu, J. H.; Pan, X. Y.; Yao, P.; Jiang, M. Langmuir 2006, 22, 2754.

26. Ye, W. J.; Leung, M. F.; Xin, J.; Kwong, T. L.; Lee, D. K. L.; Li, P. Polymer 2005, 46, 10538.

27. Sambhy, V.; MacBride, M. M.; Peterson, B. R.; Sen, A. Journal of the American Chemical Society 2006, 128, 9798. 